\title{
Scheduling the Units and Maximizing the Profit of Gencos Using LR-PSO Technique
}

\author{
B. Rampriya ${ }^{1}$ and K. Mahadevan ${ }^{2}$ \\ ${ }^{1}$ Kamaraj College of Engineering \& Technology Virudhunagar, India \\ ${ }^{2}$ Kalasalingam University, Srivilliputhur, India \\ rampriyame@gmail.com, mahadevand@rediffmail.com
}

\begin{abstract}
This paper presents the advancement in power system engineering education and research with power industry moving towards deregulation. Deregulation is a relatively recent concept, whose economic, regulatory and implementation structure continues to be adopted to the specific needs of each nation. For example, price based unit commitment in the present scenario is totally different compared to a regulated set up. Hence adequate exposes towards power engineering curriculum and new software tools are needed to support new activities in the modern power pools. This methodology performed in this will be a great challenges for the power industry and thus an individual human can take their own decision of choosing the reliable continuous supply of power from the electricity markets at an affordable price. Under this restructured system, generation companies (GENCOs) schedule their generators with the objective of maximizing their profit. The profit based unit commitment (PBUC) is performed by considering both the power and reserve generations. The quoting of power and reserve prices in spot markets and reserve markets are the important decision process. This proposed algorithm is tested for a small unit test system with 3 unit 12 hour data and the simulations are carried out to show the performance of proposed methodology using MATLAB.

Keywords: Lagrangian Relaxation (LR), Particle Swarm Optimization (PSO), Generation Companies (GENCOs), Profit based unit commitment (PBUC), Independent System Operator (ISO).
\end{abstract}

\section{Introduction}

The aim of the power generation is to predict the load demand and build the proper on/ off schedule of the generators which can minimize the total production cost. This is suitable for a vertically integrated monopolistic environment. Under deregulated environment, the on/off scheduling task by considering the power demand and spinning reserve constraints are more complex and more competitive than traditional one. Profit based unit commitment is an optimization problem to schedule generators economically together with the forecasted information such as prices and demand/reserve with the objective of maximizing the profit of individual GENCOs [1]. In this PBUC, both the power and reserve to be generated are the control variables.

There are many solution techniques such as integer programming, dynamic programming, Lagrangian relaxation and genetic algorithms are available to solve the PBUC [2-5]. Researchers also presented a review on deterministic, meta-heuristic and hybrid approaches of generation scheduling in both regulated and deregulated power markets [6]. All the above methods has their own advantages and also disadvantages.

The proposed method dealt in this research, is a hybrid LR-PSO technique and the constraints handled are power balance, unit capacity limits. This method use the advantage of PSO which can provide a near global solution combined with the advantage of LR which can find a solution within a short time.

Received: Februari 11,2010. Accepted: May 25, 2010 
B. Rampriya, et al.

\section{Profit Based Unit Commitment}

The objective of the PBUC problem is the maximization of the total profit of GENCO over the scheduling horizon. Therefore, the objective function is expressed as the difference of revenue generated and cost spent. In order to increase their own profit, GENCOs undergo the profit based unit commitment with forecasted demand, reserve and known spot prices and reserve prices in the markets[7]. Mathematically, the function is expressed as follows:

Max $P F=R V-T C$ or

Min TC-RV

Where

$P F$ is the profit of GENCO

$R V$ is the revenue of GENCO

$T C$ is the total generation cost

\section{A. Power balance constraints :}

The power balance constraint is an equality constraint that reduces the power system to a basic principle of equilibrium between total system generation and total system loads. In PBUC, GENCO can now select to sell power and reserve even below the forecasted demand and reserve level if the allocated reserve and power yields higher profit.

$$
\begin{aligned}
& \sum_{i=1}^{N} P_{i t} X_{i t} \leq D_{t}^{\prime} \quad \mathrm{t}=1,2 \ldots \mathrm{T} \\
& \sum_{i=1}^{N} R_{i t} X_{i t} \leq S R_{t}^{\prime} \quad \mathrm{t}=1,2, \ldots \mathrm{T}
\end{aligned}
$$

Where

$R_{i t}$ is reserve generation of generator $\mathrm{i}$ at time $\mathrm{t}$,

$D_{t}^{\prime}$ is forecasted demand at hour $\mathrm{t}$,

$S R_{t}^{\prime}$ is forecasted reserve at hour $\mathrm{t}$,

$P_{i t}$ is generation of unit i at time $\mathrm{t}$,

$R_{i t}$ is reserve power of unit $\mathrm{i}$ at time $\mathrm{t}$,

$X_{i t}$ is the on/off status of unit i at time t.

$T$ is the total time period

B. Unit generation and reserve limits constraints:

Generation units have lower and upper production limits for spot power and reserve power that are directly related to the machine design. These bounds can be defined as a pair of inequality constraints.

$$
\begin{aligned}
& P_{i \min } \leq P_{i t} \leq P_{i \max } \\
& 0 \leq R_{i t} \leq P_{i \max }-P_{i \min }
\end{aligned}
$$

Where

$P_{i \text { min }}$ and $P_{i \max }$ are the minimum and maximum generation of unit i.

\section{Energy Markets In Deregulated Power System}

Under open access, market driven transactions have become the new independent decision variables defining the behavior of the power systems. The goal of competitive electricity market is to enhance competition among generating utilities and bring consumer's new choices 
and economic benefits. Many power producers will sell power to customers through bilateral contracts[8] . To protect against failures of their own generators, these producers may purchase reserve power from other power producers. Reserve power is fundamentally different commodity from spot market power. The spot power price and reserve power price decisions are made based on the reserve payments made. Researchers have suggested three payment methods [9] viz., payment for power delivered , payment for reserve allocated and price process for reserve price. This research focuses only the payment for power delivered scheme.

A. Payment for power delivered

In this method, the reserve price will be paid only for the used reserve power. The reserve price is therefore higher than the spot price. Revenue and cost can calculated from

$$
\begin{aligned}
& R V=\sum_{i=1}^{N} \sum_{t=1}^{T} P_{i t} S P_{t} X_{i t}+\sum_{i=1}^{N} \sum_{t=1}^{T} r \cdot R P_{t} R_{i t} X_{i t} \\
& T C=(1-r) \sum_{i=1}^{N} \sum_{t=1}^{T}\left[F\left(P_{i t}\right)+r \sum_{i=1}^{N} \sum_{t=1}^{T} F\left(P_{i t}+R_{i t}\right)+S T\right] X_{i t} \\
& F\left(P_{i t}\right)=a_{i}+b_{i} P_{i}+c_{i P i}^{2} \quad \$ / h r
\end{aligned}
$$

Where

$S P_{t}$ is the forecasted price at hour $\mathrm{t}$,

$R P_{t}$ is the forecasted reserve price at hour $\mathrm{t}$,

$r$ is the probability that the reserve is called and generated,

$F_{i}$ is the fuel cost function of generator $\mathrm{i}$,

$S T$ is the start up cost.

$F\left(P_{i t}\right)$ is the fuel cost equation of generator $i$ at time t.

$a_{i}, b_{i}$ and $c_{i}$ are the cost coefficients of generator

\section{Hybrid Solution Methodology}

A. Formation of LagrangianFunction

The LR procedure solves the UC problem by relaxing coupling constraints into objective function by using Lagrangian multipliers. The Lagrangian function is given by

$$
\begin{aligned}
& (1-r) \sum_{i=1}^{N} \sum_{t=1}^{T}\left[F\left(P_{i t}\right)+r \sum_{i=1}^{N} \sum_{t=1}^{T} F\left(P_{i t}+R_{i t}\right)+S T\right] X_{i t} \sum_{i=1}^{N} \sum_{t=1}^{T} P_{i t} S P_{t} X_{i t i t} \\
& \sum_{i=1}^{N} \sum_{t=1}^{T} r \cdot R P_{t} R_{i t} X_{i t} \\
& -\sum_{t=1}^{T} \lambda\left(D_{t}^{\prime}-\sum_{i=1}^{N} P_{i t} X_{i t}\right)-\sum_{t=1}^{T} \mu\left(S R_{t}^{\prime}-\sum_{i=1}^{N} R_{i t} X_{i t}\right)
\end{aligned}
$$

The relaxed problem is then decomposed into sub problems for each unit. The Lagrangian function can be modified after neglecting the constant terms can be rewritten as $L$ :

$$
\sum_{i=1}^{N}\left[\sum_{t=1}^{T}\left\{(1-r) F\left(P_{i t}\right)+r F\left(P_{i t}+R_{i t}\right)+S T-P_{i t} \cdot S P_{t} r \cdot R P_{t} \cdot R_{i t}+\lambda_{t} P_{i t}+\mu R_{i t}\right\} X_{i t}\right]
$$




\section{B. Dynamic Programming Part}

The constrained minimum of Lagrangian function $L$ for each unit is solved to obtain $P_{i t}$ and $\mathrm{R}_{i t}$ for $\mathrm{t}=1 \ldots \mathrm{T} ; i=1 \ldots N$; using two-state dynamic programming. The objective of this problem is to minimise the dual problem $\mathrm{q}(\lambda, \mu)$.

The function to be minimized is

$$
K=(1-r) F\left(P_{i t}\right)+r F\left(P_{i t}+R_{i t}\right)-P_{i t} S P_{t}-r R P_{t} R_{i t}+\lambda_{t} P_{i t}+\mu_{t t}^{\prime} R_{i t}
$$

The minimum of the function is found by taking the first derivative of $\mathrm{K}$ and setting it to zero.

$$
\begin{aligned}
& \frac{\partial K}{\partial P_{i t}}=0 \\
& \frac{\partial K}{\partial R_{i t}}=0
\end{aligned}
$$

The solution of this equation is

$$
P_{i t}=\frac{1}{1-r}\left(A_{i t}-r B_{i t}\right)
$$

$$
R_{i t}=\frac{1}{1-r}\left(-A_{i t}+B_{i t}\right)
$$

Where $A_{i t}=\frac{S P_{t}-\lambda_{t}-b_{i}}{2 a_{i}}$

$$
B_{i t}=\frac{r \cdot R P_{t}-\mu_{t}-r \cdot b_{i}}{r .2 a_{i}}
$$

After obtaining the solution,dual value $\mathrm{q}$ is calculated as

Min

$$
\mathrm{q}(\lambda, \mu)=\sum_{i=1}^{N} \min \left[\sum_{t=1}^{T}\left\{(1-r) F\left(P_{i t}\right)+r F\left(P_{i t}+R_{i t}\right)+S T-P_{i t} \cdot S P_{t} r \cdot R P_{t} \cdot R_{i t}+\lambda_{t} P_{i t}+\mu R_{i t}\right\} X_{i t}\right]
$$

\section{Updating Lagrangian Multipliers}

In this research, Lagrangian multipliers are then updated using PSO to overcome the difficulties of GA[2]. LR method can provide a fast solution but the quality of solution strongly depends on the algorithm used to update the Lagrangian multipliers. Particle swarm optimization (PSO) is a population based stochastic optimization technique developed by Dr. Eberhart and Dr. Kennedy in 1995, inspired by social behavior of bird flocking or fish schooling. The system is initialized with a population of random solutions and searches for optima by updating generations. However, unlike GA, PSO has no evolution operators such as crossover and mutation.

In PSO, the potential solutions, called particles, fly through the problem space by following the current optimum particles. The PSO model consists of swarm of particles moving in $\mathrm{d}-$ dimensional real valued space of possible solutions[10-12]. Each particle has a position $X_{i}=\left(x_{i 1}\right.$, $\left.x_{i 2}, . . x_{i d}\right)$ and a velocity of $V_{i}=\left(v_{i 1}, v_{i 2}, . . v_{i d}\right)$. Each particle has its own best position as $P_{i}=\left(p_{i 1}\right.$, $\left.p_{i 2}, . . p_{i d}\right)$ and a global best position $P_{g}=\left(P_{g 1}, P_{g 2}, \ldots P_{g d}\right)$ obtained through communication with it fellow neighbor particles.

For each iteration, the velocity is updated and the particle is moved to new position[10] . This new position is simply calculated as the sum of previous position and the new velocity:

$$
x_{i k}{ }^{r+1}=x_{i k}{ }^{r}+v_{i k}{ }^{r+1} \quad i=1,2 \ldots n ; \quad k=1,2, . . d
$$


The update of the velocity from the previous velocity to the new velocity is determined by :

$$
v_{i k}^{r+1}=w v_{i k}^{r}+c_{1} \operatorname{rand1}(0,1)\left(p_{i k}-X_{i k}^{r}\right)+c_{2} \operatorname{rand} 2(0,1)\left(p_{g k}-X_{i k}^{r}\right)
$$

where

$r$ is the iteration count

$c_{1}, c_{2}$ are the acceleration constant

rand1 $(0,1)$ and rand2 $(0,1)$ are the uniform random value between $[0,1]$.

$w$ is the inertia weight factor and is updated by

$w(k)=w_{\max }-\left(w_{\max }-w_{\min }\right) * k / k_{\max }$

$k$ is the current number of iterations

$k_{\text {max }}$ is the maximum number of iterations.

$w_{\text {min }}$ is the initial inertia weight

$w_{\text {max }}$ is the inertia weight in the last iteration.

\section{Fitness Function}

Frame the dual value $\mathrm{q}$ by the equation (8). The value of $\mathrm{q}$ indicates the fitness of the candidate solution of each individual.

\section{E. Primal Function}

Obtain the values of $P_{i t} *$ and $R_{i t} *$ by solving economic dispatch for each hour with the $X_{i t}$ obtained from the winning population. The primal value $J$ with the values of $P_{i t}{ }^{*}$ and $R_{i t}{ }^{*}$.

\section{G. Terminating Criteria}

The difference between the primal and dual values is used as a terminating criteria. Duality gap is given by $\varepsilon=\frac{J-q}{|J|}$ which is the terminating criteria for the proposed methodology. The overall procedure is illustrated in fig. 1 as the flowchart given below:

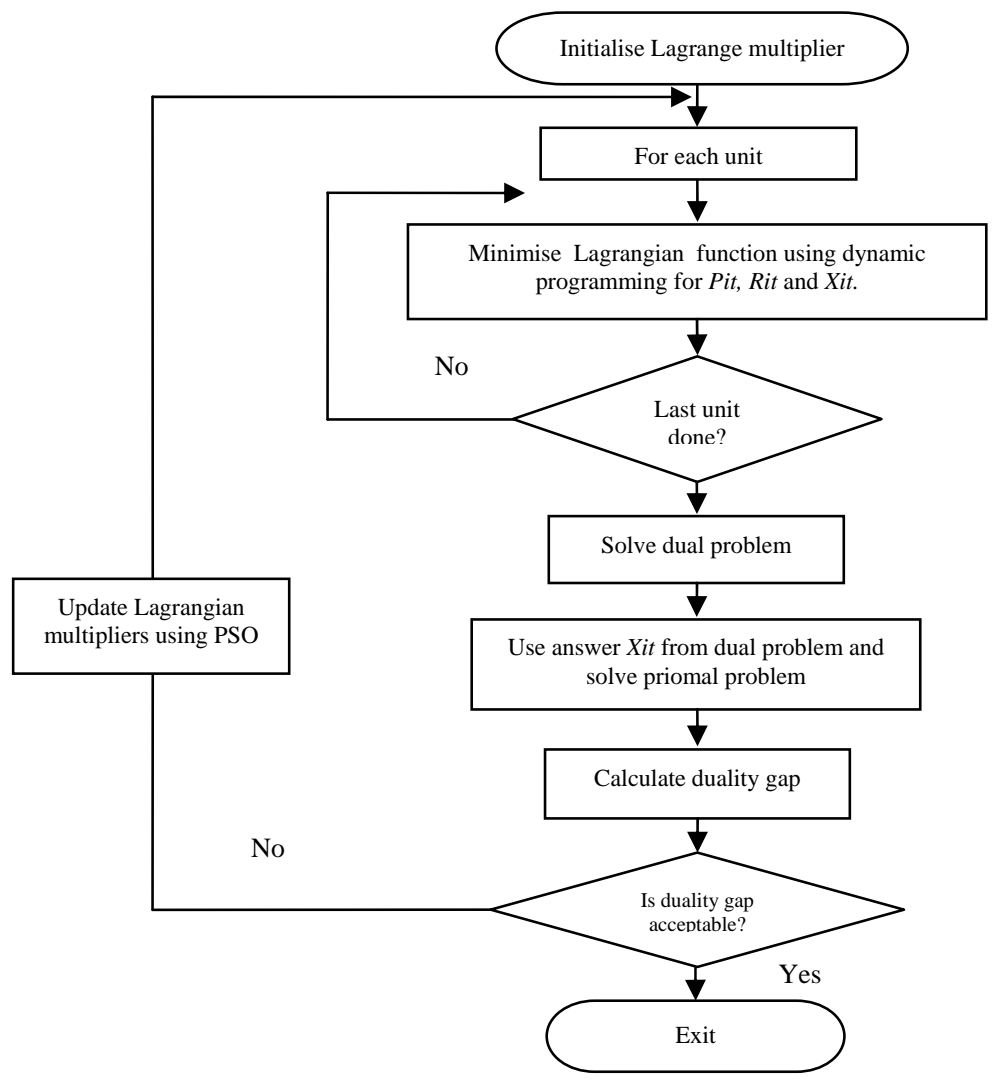

Figure 1. Flowchart of LR-PSO method 
B. Rampriya, et al.

\section{Simulation Results}

Simulations are carried out for the test system with 3 unit 12 period system. The unit data and forecasted demand and reserve data of this test system are given in Table 1 and Table 2. The proposed methodology is implemented on INTEL[R], Pentium [R] CPU 2 GHZ, 1GB RAM and simulated in MATLAB environment.

The effect of probability that reserve is called and generated $(r)$ is investigated and kept as 0.005 . Here, the reserve price is fixed at the triple times of spot price for payment for power delivered method. Table 3 shows the primal value (J), dual value (q) and the terminating criteria duality gap. It is clear from Table 1 that at the end of seventh iteration, the duality gap is reduced to 0.0294 .

Table 4 shows the power and reserve allocation for the same test system by LR-PSO. In PBUC, GENCO can now select to sell power and reserve even below the forecasted level if the allocated reserve and power yields higher profit. According to this allocation schedule, the power and reserve are distributed by satisfying the capacity constraints and also the profit has also been maximized by LR-PSO as 9465.6\$. The results of Muller method [13] and hybrid methods such as LR-gradient search, LR-EP[1] and LR-PSO for the same test system can be compared and tabulated as shown in Table 5.

Table 1. Unit Data ( 3 Unit, 12 Hour System)

\begin{tabular}{lccc}
\hline & Unit I & Unit II & Unit III \\
\hline Pmax (MW) & 600 & 400 & 200 \\
\hline Pmin (MW) & 100 & 100 & 50 \\
\hline $\mathrm{a}(\$ / \mathrm{hr})$ & 500 & 300 & 100 \\
\hline $\mathrm{b}(\$ / \mathrm{hr})$ & 10 & 8 & 6 \\
\hline $\mathrm{c}(\$ / \mathrm{hr})$ & 0.002 & 0.0025 & 0.005 \\
\hline Min up time(h) & 3 & 3 & 3 \\
\hline Min down time $(\mathrm{h})$ & 3 & 3 & 3 \\
\hline Start up cost $(\$)$ & 450 & 400 & 300 \\
\hline Initial status(h) & -3 & 3 & 3 \\
\hline
\end{tabular}

Table 2. Forecasted Demand and Reserve Data For 12 Hour

\begin{tabular}{cccc}
\hline Hour & $\begin{array}{c}\text { Forecasted } \\
\text { demand } \\
(\mathrm{MW})\end{array}$ & $\begin{array}{c}\text { Forecasted } \\
\text { reserve } \\
(\mathrm{MW})\end{array}$ & $\begin{array}{c}\text { Forecasted } \\
\text { spot price } \\
(\mathbf{M} / \mathbf{M H})\end{array}$ \\
\hline 1 & 170 & 20 & 10.55 \\
\hline 2 & 250 & 25 & 10.35 \\
\hline 3 & 400 & 40 & 9.00 \\
\hline 4 & 520 & 55 & 9.45 \\
\hline 5 & 700 & 70 & 10.00 \\
\hline 6 & 1050 & 95 & 11.25 \\
\hline 7 & 1100 & 100 & 11.30 \\
\hline 8 & 800 & 80 & 10.65 \\
\hline 9 & 650 & 65 & 10.35 \\
\hline 10 & 330 & 35 & 11.20 \\
\hline 11 & 400 & 40 & 10.75 \\
\hline 12 & 550 & 55 & 10.60 \\
\hline
\end{tabular}


Table 3. Duality Gap For 3-Unit, 12 Hour System Using LR-Gradient Search Method Iteration Dual Primal Duality

Function(\$) function (\$) gap

\begin{tabular}{cccc}
\hline 1 & 0 & 100000 & infinity \\
\hline 2 & 42757 & 99531 & 1.3278 \\
\hline 3 & 51174 & 81426 & 0.5912 \\
\hline 4 & 54346 & 65922 & 0.2130 \\
\hline 5 & 55397 & 65468 & 0.1818 \\
\hline 6 & 54782 & 56707 & 0.0351 \\
\hline 7 & 55071 & 56692 & 0.0294 \\
\hline 8 & 54937 & 57677 & 0.0499 \\
\hline 9 & 54967 & 56708 & 0.0317 \\
\hline 10 & 54664 & 57480 & 0.0515 \\
\hline
\end{tabular}

Table 4. Power and Reserve Allocation For 3-Unit, 12 Hour System Using LR-PSO ( $r=0.005$ \& Reserve Price $=3 *$ Spot Price)

\begin{tabular}{ccccccc}
\hline \multirow{2}{*}{ Hour } & \multicolumn{3}{c}{ Power (MW) } & \multicolumn{4}{c}{ Reserve (MW) } \\
\cline { 2 - 7 } & U1 & U2 & U3 & U1 & U2 & U3 \\
\hline 1 & 0 & 0 & 170 & 0 & 0 & 0 \\
\hline 2 & 0 & 0 & 200 & 0 & 0 & 0 \\
\hline 3 & 0 & 0 & 200 & 0 & 0 & 0 \\
\hline 4 & 0 & 0 & 200 & 0 & 0 & 0 \\
\hline 5 & 0 & 380 & 200 & 0 & 20 & 0 \\
\hline 6 & 0 & 400 & 200 & 0 & 0 & 0 \\
\hline 7 & 0 & 400 & 200 & 0 & 0 & 0 \\
\hline 8 & 0 & 400 & 200 & 0 & 0 & 0 \\
\hline 9 & 0 & 400 & 200 & 0 & 0 & 0 \\
\hline 10 & 0 & 130 & 200 & 0 & 35 & 0 \\
\hline 11 & 0 & 200 & 200 & 0 & 40 & 0 \\
\hline 12 & 0 & 350 & 200 & 0 & 50 & 0 \\
\hline
\end{tabular}

Table 5. Profit of Genco For 3-Unit, 12 Hour System Using LR-PSO

\begin{tabular}{cccc}
\hline Hour & Revenue (\$) & Cost(\$) & Profit(\$) \\
\hline 1 & 1793.5 & 1264.5 & 529.0 \\
\hline 2 & 2070 & 1500 & 570.0 \\
\hline 3 & 1800 & 1500 & 300.0 \\
\hline 4 & 1890 & 1500 & 390.0 \\
\hline 5 & 5803 & 5202 & 601.0 \\
\hline 6 & 6756.8 & 5401.6 & 1348.4 \\
\hline 7 & 6788.5 & 5402.1 & 1377.9 \\
\hline 8 & 6395.6 & 5401.4 & 988.6 \\
\hline 9 & 6214.7 & 5401.2 & 808.8 \\
\hline 10 & 3701.9 & 2883.8 & 818.1 \\
\hline 11 & 4306.4 & 3501.8 & 804.6 \\
\hline 12 & 5838 & 4908.7 & 929.2 \\
\hline
\end{tabular}


B. Rampriya, et al.

\begin{tabular}{ccc}
\hline \multicolumn{2}{c}{ Total profit(\$) } & 9465.6 \\
\hline Table 6. & Comparison of Methods For 3-Unit, 12 Hour System \\
\hline Method & Profit(\$) \\
\hline LR-gradient search & 8672.35 \\
\hline Muller method & $9056.49[13]$ \\
\hline LR-EP & $9074.3[1]$ \\
\hline LR-PSO & 9465.6 \\
\hline
\end{tabular}

Table 7. Summary Of Parameters Selected

\begin{tabular}{cc}
\hline Parameters & 3 unit $\mathbf{~ 1 2}$ hour system \\
\hline Population size & 50 \\
\hline No. of iterations & 25 \\
\hline$w_{\max }$ & 0.9 \\
\hline$w_{\min }$ & 0.4 \\
\hline$C_{1}$ and $c_{2}$ & 2 \\
\hline
\end{tabular}

\section{Conclusion}

Application of PSO to update the Lagrangian multipliers is an effective technique to solve unit commitment problem. This hybrid technique used the advantage of LR, which can handle various constraints and provides a faster solution. At the same time, the global-search property of PSO was included into the method, and used to update the Lagrange multipliers, thereby improving the performance of traditional LR method. This LR-PSO algorithm produces results better than other methods specified. The results obtained show that the proposed method is simple, efficient and have great potential for solving practical UC problems. This proposed method helps generation company to make decision, how much power and reserve should be sold in markets and how to schedule generators in order to receive the maximum profit.

The research can be extended by varying the probability that the reserve can be called and also by varying the reserve price in terms of spot price. The proposed hybrid methodology can be incorporated on PBUC problem to impose the ramp rate, environmental emission and security constraints [14 -16] .The PBUC problem can also be solved using Multi agent system which utilizes the rule based and dynamic programming methods to sole the new optimization problem corporately, and they could be located on different computers in the same computer network anywhere[17].

\section{References}

[1] Pathom Attaviriyanupap, Hiroyuki kita, Jun Hasegawa, "A Hybrid LR-EP for solving new profit based UC problem under competitive environment”, IEEE Transactions on Power Systems, Vol. 18, No. 1, February 2003, 229-236.

[2] Charles W. Ritcher, Gerald B. Sheble "A Profit based unit commitment GA for the competitive environment," IEEE Transactions on Power Systems, Vol. 15, No. 2, May 2000, 715-721.

[3] N. P. Padhy, "Unitcommitment-A Bibliographical Survey", IEEE Transactions on Power Systems, Vol. 19, No. 2, May 2004, 1196-1205.

[4] H. Y. Yamin , S. M. Shahidehpour, "Unit commitment using a hybrid model between Lagrangian relaxation and genetic algorithm in competitive electricity markets", Electric power Systems Research 68(2004), 83-92.

[5] Chuan-Ping Cheng, Chih-Wen Liu and Chun-Chang Liu, "Unit commitment by Lagrangian Relaxation and genetic algorithms", IEEE Transactions on Power Systems, Vol. 15, No. 2, May 2000, 707-714.

[6] N. P. Padhy, "Unit commitment- problem under deregulated environment- A Review", IEEE Transactions on Power Systems, 2003,1088-1094. 
[7] Pathom Attaviriyanupap, Hiroyuki kita, Eiichi Tanaka and Jun Hasegawa , "New Bidding Strategy Formulation For Day-Ahead Energy and Reserve markets Based on Evolutionary Programming”, International Journal of Electrical power \& Energy Systems, Vol . 27, No. 3 pp. 157-167, March 2005.

[8] S. M. Shahidehpour, H. Y. Yamin, Z. Li, "Market operations in Electric power systems (February),” John Wiley \& Sons, 2002.

[9] Eric H.allen, Marija D. Ilic, "Reserve markets for power system reliability", IEEE Ttransactions on power systems, Vol. 15, No. 1, February 2000, 228-233.

[10] Y. H. Shi, R. C. Eberhart, “A Emprical study of particle swarm optimization,” in Proc. Conf. Evolutionary computation, Wahington, DC, Jul.6-9,1999.

[11] T. O. Ting, M. V. C. Rao , C. K. Loo, “A Novel approach for unit commitment problem via an effective hybrid particle swarm optimization," IEEE transactions on power systems, Vol. 21, No. 1, February, 2006,411-418.

[12] M. Clerc , J. Kennedy, ” The particle swarm: Explosion stability and convergence in a multi-dimensional complex space, "IEEE Transactions on Evolutionaruy computation, Vol. 6, No. 1, February, 2002, 58-73.

[13] K. Chandram, N. Subrahmanyam, "Improved pre-prepared power demand table with Muller method for solviong profit based unit commitment”, IEEE conference TENCON, November 2008.

[14] C. Wang \& S. M. Shahidehpour, "Effects of ramp limits on unit commitment and economic dispatch”, IEEE Transactions on power systems, 1993, 1341-1350.

[15] Shih-Yih Lai and Ross Bldrick, "Unitcommitment with Ramp Multipliers”, IEEE Trans. On Power systems, Vol. 14, No1, Feb. 1999, pp.59-63.

[16] H. Y. Yamin, "Security Constrained Price based Unit Commitment in the Deregulated Power Market”, IEEE Large Engineering system conference proceedings, 2002.

[17] Jing Yu, Jianzhong Zhou, Wei Wu, and Junjie Yang, "Solution of profit based unitcommitment problem by using mutli agent system”, IEEE conference Proceedings of $5^{\text {th }}$ Wold Congress on Intelligent Control and Automation, June 15-19, 2004.

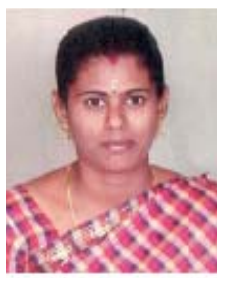

C. Rampriya is an Assistant Professor at Kamaraj College of engineering and technology. She received her B.E. and M.E. degrees in Electrical and Electronics and Power systems engineering from Manonmaniam sundaranar university and Anna university in 2000 and 2004, respectively. She is an active member in Indian society for technical education. She has presented various paper in National conference and international conference. Her area of interest includes power system optimization, Evolutionary computation techniques.

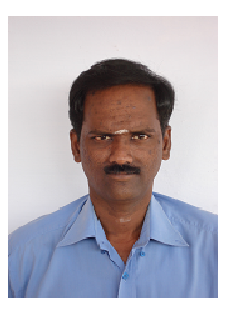

K. Mahadevan was born in Tirumangalam, India, in 1968. He graduated in Electrical and Electronics Engineering and post graduated in Industrial Engineering from Madurai Kamaraj University, India in 1993 and 1997 respectively. He was awarded with Ph.D degree from the same university in the year 2006. From 1997 onwards he has been a faculty member of Electrical Engineering at Kalasalingam University, Krishanakoil, India. His fields of interest include Power system Operation \& Control, Power System Optimization and Evolutionary Computation. 